\title{
Pitch strength and Stevens's power law
}

\author{
WILLIAM P. SHOFNER and GEORGE SELAS \\ Loyola University Chicago, Chicago, Illinois
}

\begin{abstract}
Recent studies have suggested that the saliency or the strength of pitch of complex sounds can be accounted for on the basis of the temporal properties in the stimulus waveform as measured by the height of the first peak in the waveform autocorrelation function. We used a scaling procedure to measure the pitch strength from 15 listeners for four different pitches of complex sounds in which the height of the first peak in the autocorrelation function systematicallyvaried. Pitch strength judgments were evaluated in terms of a modification of Stevens's power law in which temporal information was used from both the waveform fine structure and the envelope. Best fits of this modified power law to the judged pitch strengths indicate that the exponent in Stevens's power law is greater than 1 . The results suggest that pitch strength is primarily determined by the waveform fine structure, but the stimulus envelope can also contribute to the pitch strength.
\end{abstract}

S.S. Stevens (1975) described two classes of sensory continua-namely, a metathetic or qualitative continuum and a prothetic or quantitative continuum. The prothetic continuum is related to the intensity of the stimulus and can be scaled by human observers with the use of techniques such as magnitude estimation. The relationshipbetween the magnitude of the sensation $(\psi)$ and the intensity of the stimulus dimension $(\phi)$ has been studied for a variety of stimuli (see S. S. Stevens, 1975) and can be described mathematically by a power function having the form

$$
\psi=k \phi^{\beta},
$$

where $k$ is a constant. This power function is generally known as Stevens's power law. Most importantly, the exponent, $\beta$, is a characteristic of any given prothetic continuum and indicates how fast the magnitude of the sensation grows as the stimulus intensity increases. For example, in the auditory system, the perceptual continuum of loudness has often been studied in relation to Stevens's power law, typically yielding $\beta<1$ (e.g., Hellman, 1976, 1981; Logue, 1976; J. C. Stevens \& Guirao, 1964; S. S. Stevens, 1955, 1975). Thus, the loudness of a sound increases at a slower rate of change than does the sound pressure level; that is, the loudness function is a compressive function.

Although the pitch of complex sounds does not exist on a prothetic continuum, the saliency or strength of pitch can be scaled on a quantitative continuum with techniques such as magnitude estimation. Pitch strength is a perception that is independent of pitch per se and refers to how

This research was supported by Program Project Grant P01 DC00293 from the National Institute of Deafness and Communication Disorders. G.S. was supported by the Mie Kim Najita Summer Fellowship. We thank W. A. Yost and C. B. Braun for their helpful suggestions. We also thank the reviewers for their comments and criticisms. Correspondence concerning this article should be addressed to W. P. Shofner, Parmly Hearing Institute, Loyola University Chicago, 6525 N. Sheridan Rd., Chicago, IL 60626 (e-mail: wshofne@luc.edu). strong a listener perceives the sensation of pitch to be. For example, consider two complex sounds of equal loudness that both produce a pitch corresponding to $250 \mathrm{~Hz}$. One of these sounds may have a strong pitch at $250 \mathrm{~Hz}$, whereas the other may have a faint or weak pitch at $250 \mathrm{~Hz}$. Fastl and Stoll (1979) used a magnitude estimation procedure to scale the pitch strength for a variety of stimuli that produce the perception of pitch. Although listeners generally rated stimuli characterized by line spectra as having greater pitch strength than stimuli characterized by continuous spectra, these authors did not relate pitch strength to any specific stimulus feature or dimension in a quantitative manner. Indeed, unlike loudness, which is clearly related to the overall sound pressure level, the stimulus dimension related to pitch strength is more equivocal. More recently, however, it has been argued that pitch strength can be predicted on the basis of the height of the first peak (AC1) in the stimulus autocorrelation function (Patterson, Handel, Yost, \& Datta, 1996; Yost, 1996b; Yost, Patterson, \& Sheft, 1996). Yost (1996b) scaled the pitch strengths of iterated rippled noises and described the relationship between $\mathrm{AC} 1$ and the perceived pitch strength with an exponential function having the form

$$
\psi=a+\left(10^{b A C 1}\right)
$$

where $a=10$ and $b=2$.

In neither of the studies above was pitch strength evaluated in terms of Stevens's power law. Based on the shape of the exponential function described by Yost (1996b), it would be predicted that $\beta$ in Stevens's power law would be greater than 1 if $\mathrm{AC} 1$ were equivalent to the stimulus dimension $\phi$. Thus, if $\beta$ is greater than 1, pitch strength will increase at a faster rate of change than will the height of AC1. Moreover, if pitch strength is related to the height of $\mathrm{AC} 1$, as described by Stevens's power law, it would be predicted that there should be no effect of relative starting phase on the pitch strengths for harmonic tone complexes. Harmonic tone complexes in which all components are 
added in cosine-starting phase have the same waveform autocorrelation function as harmonic tone complexes that are composed of the same frequency components, but added in random phase (see Figure 1). However, it is known that random-phase and cosine-phase harmonic tone complexes can be discriminated from one another (see Patterson, 1987), presumably because the perceived pitch strength of a random-phase complex tone is weaker than that of the corresponding cosine-phase complex tone. One difference between cosine-phase and random-phase complex tones is found in the stimulus envelopes (see Figure 1); cosine-phase complex tones have highly modulated or "peaky" envelopes, whereas random-phase tone complexes have "flatter" envelopes. Thus, the stimulus envelope may also be a feature related to pitch strength. Presumably, the perceived pitch strength of a random-phase tone complex would be weaker than that of the cosinephase tone complex owing to the smaller AC1 observed in the random-phase envelope.

In the present study, pitch strength was measured from human listeners for harmonic complex tones and iterated rippled noises. Iterated rippled noises were used as test stimuli because they have become an important class of stimuli for studying pitch perception. The stimuli were chosen so that there was a continuum in pitch strength but with minimal changes in timbre. Specifically, we were interested in the application of Stevens's power law to the continuum of pitch strength, and we suggest that pitch strength can best be accounted for by using a modification to Stevens's power law.

\section{METHOD}

\section{Listeners}

The listeners in this experiment were 15 undergraduate students between the ages of $18-45$ years (median age, 20 years). ${ }^{1}$ The listeners were paid and were participating in other psychophysical experiments. The listeners sat in an IAC single-walled sound-attenuating chamber and listened to stimuli diotically over Sennheiser HD 520 II headphones.

\section{Stimuli}

The 10 test stimuli consisted of cosine-phase harmonic complex tones, random-phase harmonic complex tones, seven different infinitely iterated rippled noises (IIRN), and wideband noise (WBN). IIRN is generated when the WBN is delayed, attenuated, and the delayed version of the noise is added to the original WBN through a positive feedback circuit (Figure 1; see also Shofner \& Yost, 1995; Yost, 1996a). The pitch of iterated rippled noise is matched to a frequency of 1/delay (Yost, 1996a). There were a total of four different pitch conditions in which the periods of the complex tones and delays of IIRN were 2, 4, 8, and $16 \mathrm{msec}$. The fundamental frequencies ( $f 0)$ and corresponding matched pitches were 500, 250, 125, and $62.5 \mathrm{~Hz}$, respectively. For every stimulus, the autocorrelation functions of both the waveform and the envelope were determined. The stimulus envelopes were extracted by using the Hilbert transform. Figure 1 shows examples of waveform and envelope autocorrelation functions for stimuli having a period or delay of $8 \mathrm{msec}$. The bottom right-hand panel in Figure 1 shows the height of $\mathrm{AC} 1$ of the waveform and the envelope for each of the 8-msec test stimuli used in the present study. The height of $\mathrm{AC} 1$ is a measure of the periodicity at that particular time lag. ${ }^{2}$ The waveform amplitudes of all test stimuli had equal rms amplitudes.
WBN was generated with a Wavetek VCG/Noise generator (Model 132) with parameters set to yield a pseudo-random noise having a $10-\mathrm{kHz}$ bandwidth that repeated itself every $6.55 \mathrm{sec}$. The output of the noise generator was divided into two channels; one channel was delayed through an Eventide Precision Delay Line (Model PD 860). The outputs of the two channels were lowpass filtered separately at a cutoff frequency of $15 \mathrm{kHz}$ (FT5 Tucker-Davis Technologies). The attenuation of the delayed channel was determined by a programmable attenuator (PA4 Tucker-Davis Technologies), and the two channels were then summed together (SM3 Tucker-Davis Technologies) to yield an IIRN. Casual listening to IIRN for a fixed delay indicates that pitch strength decreases as the amount of delayed attenuation in the circuit increases. The seven IIRN stimuli had delayed attenuations of $-1,-2,-3,-4,-5,-6$, and $-8 \mathrm{~dB}$. For WBN and each IIRN, $5 \mathrm{sec}$ of the waveforms were sampled at $50 \mathrm{kHz}$ and stored on disk as stimulus files. If the test stimuli were either WBN or an IIRN, a random 500-msec sample was extracted from the 5-sec sample for presentation on a trial.

Harmonic tone complexes were generated on a digital array processor (AP2 Tucker-Davis Technologies) and were stored on disk as stimulus files. Harmonic tone complexes were composed of the $f 0$ and all higher harmonics up to and including $10 \mathrm{kHz}$. Consequently, the $500-\mathrm{Hz}$ harmonic tone complexes (2-msec period) were composed of 20 components, the $250-\mathrm{Hz}$ harmonic tone complexes (4-msec period) were composed of 40 components, the $125-\mathrm{Hz}$ harmonic tone complexes (8-msec period) were composed of 80 components, and the $62.5-\mathrm{Hz}$ harmonic tone complexes (16-msec period) were composed of 160 components. Thus, the overall bandwidth of the tone complexes were similar to the bandwidths of the WBN and IIRN stimuli, which also contained frequencies of up to $10 \mathrm{kHz}$. Since all stimuli contained frequencies covering a similar range, timbre changes across stimuli of a fixed $f 0$ should be minimal (e.g., Singh \& Hirsh, 1992). All frequency components were of equal amplitude and added in cosine-starting phase or random-starting phase. For the randomphase condition, a new random-phase harmonic tone complex was generated prior to the start of each block of trials.

Stimulus presentation and data acquisition were controlled by a Gateway computer system (E-4200) with Tucker-Davis Technologies (TDT) modules. The stimuli were played through a D/A converter (TDT DD1) at a conversion rate of $50 \mathrm{kHz}$, lowpass filtered at $15 \mathrm{kHz}$ (TDT PF1), attenuated (TDT PA4), and amplified (TDT HB6). The overall level was $73 \mathrm{~dB}$ SPL. The listeners entered their magnitude judgments of pitch strength on the computer keyboard.

\section{Procedure}

On a given trial, three different stimuli were presented. The first stimulus was a $10-\mathrm{kHz}$ WBN; the second stimulus was the test sound used to judge pitch strength; the third stimulus was a harmonic tone complex with all components added in cosine-starting phase. The listeners were instructed that the first stimulus had 0-pitch strength and that the third stimulus had the maximum pitch strength of 100 . If the second stimulus had a pitch strength equal to the first stimulus, the pitch strength of the second stimulus should be assigned a rating of 0 , and if the second stimulus had a pitch strength equal to the third stimulus, the pitch strength of the second stimulus should be assigned a rating of 100 . If the second stimulus had a pitch strength between that of the first and third stimuli, the pitch strength of the second stimulus should be assigned a rating that is scaled relative to the pitch strengths of the first and third stimuli. This procedure is identical to that used by Yost (1996b). If needed, the listeners could listen to any trial repeatedly until they felt confident enough to assign a rating to the perceived pitch strength. All stimuli had a duration of $500 \mathrm{msec}$ with $10-\mathrm{msec} \cos ^{2}$ rise/fall times. The time between successive stimuli was $500 \mathrm{msec}$.

During a test session, the listeners typically ran two conditions and were given a break after every four blocks. Each block consisted of 40 trials of 10 different test stimuli having the same pitch but different pitch strength. The pitches of stimuli were not varied within a block of trials, because we were not interested in whether there exists an ef- 
Waveform Autocorrelation Functions

cosine-phase tone complex

(cos)

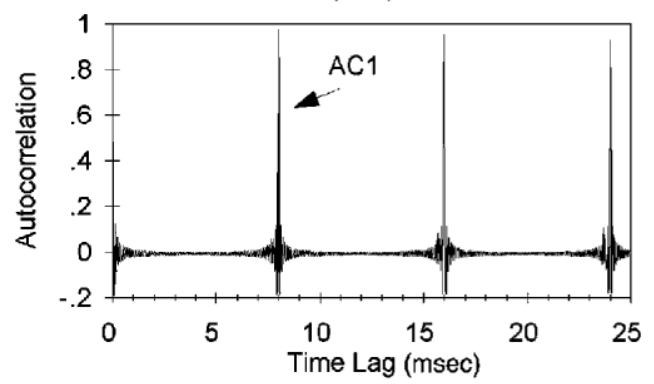

random-phase tone complex

(rnd)

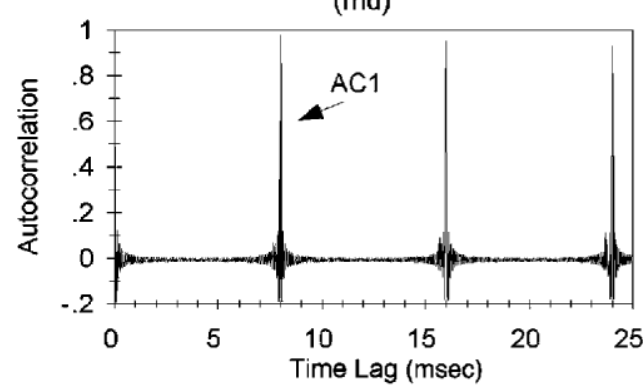

infinitely iterated rippled noise
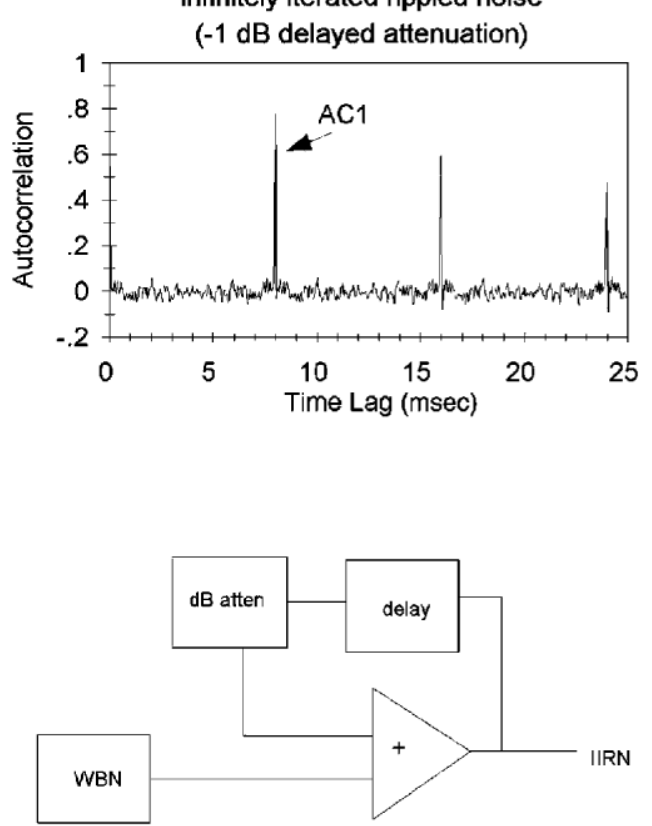

Envelope Autocorrelation Functions

cosine-phase tone complex

(cos)

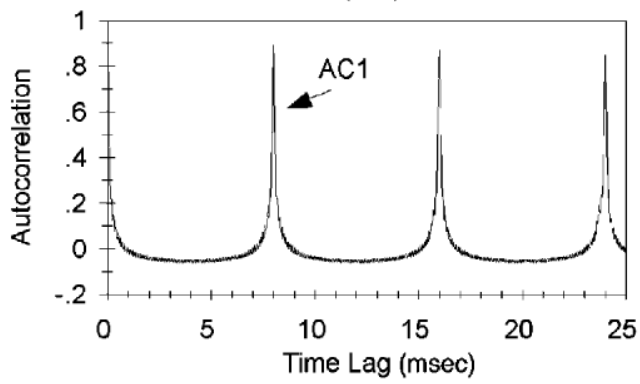

random-phase tone complex

(md)

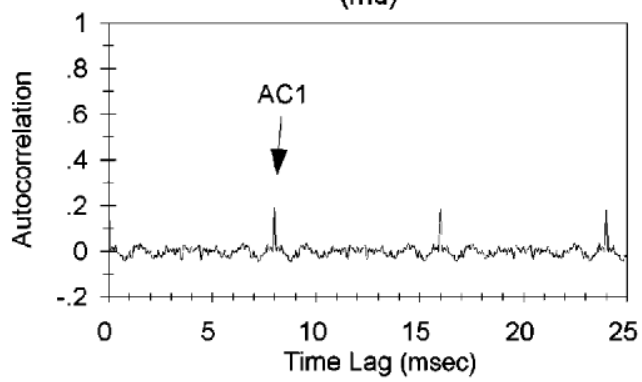

infinitely iterated rippled noise

( $-1 \mathrm{~dB}$ delayed attenuation)
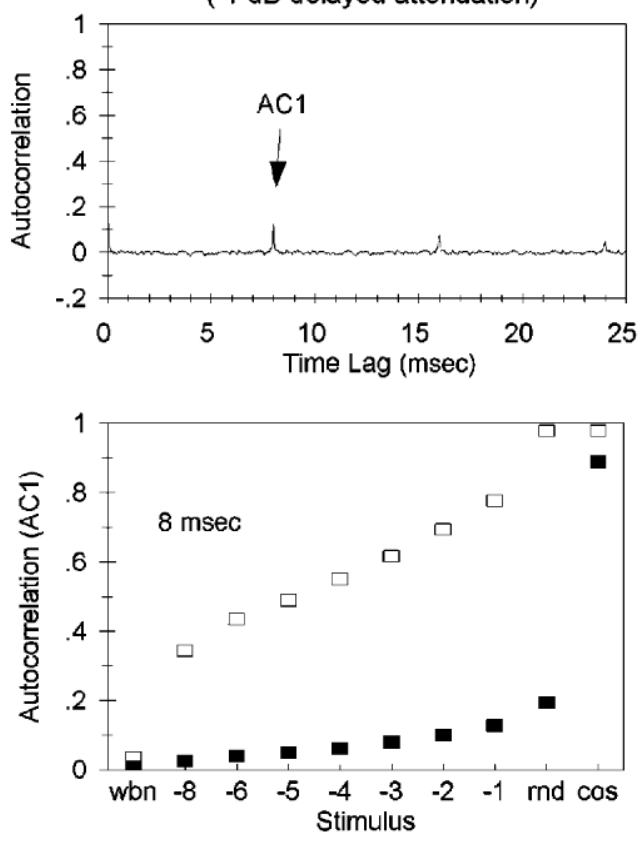

$\square$ waveform $\mathbf{a}$ envelope

Figure 1. Examples of the autocorrelation functions for stimulus waveforms (left-hand column) and stimulus envelopes (right-hand column). The cosine-phase and random-phase tone complexes have periods of $8 \mathrm{msec}$, and the IIRN has a delay of $8 \mathrm{msec}$ and a delayed attenuation of $-1 \mathrm{~dB}$. The first peaks in the autocorrelation functions (AC1) are indicated by the arrow in each panel. The bottom right-hand panel shows the height of the AC1s of the waveforms (open squares) and of the envelopes (filled squares) for each of the 10 test stimuli. In this and subsequent figures, cos and rnd represent cosine-phase and random-phase harmonic tone complexes, respectively; wbn represents wideband noise; IIRN stimuli are represented by the numbers -1 through -8 , which indicate the values of the delayed attenuation in decibels. The bottom left-hand panel shows a schematic circuit for generating IIRN. 
fect of pitch per se on pitch strength. Fixing the pitch of the stimuli within a given block of trials is identical to the procedure previously used by Fastl and Stoll (1979). Each of the 10 test stimuli was presented randomly four times during a block of trials. Included among the 10 test stimuli were WBN and cosine-phase harmonic complex tones (see Figure 1). Most of the listeners (12/15) completed a total of 10 blocks for each of the four conditions, yielding judgments of pitch strength based on 40 trials for each test stimulus. Three listeners completed a total of 10 blocks for three of the four conditions, but each had one condition in which a total of 9 blocks were completed; for these cases, judgments of pitch strength for each test stimulus were based on 36 trials rather than on 40 trials. The final magnitude judgment for each stimulus was determined as the average judgment of the 36-40 trials.

\section{Results}

Figure 2 shows examples of the magnitude judgments of pitch strength for individual listener J.D. for the four conditions studied. The open circles show the average magnitude judgments for each of the various test stimuli (see Figure 1). For each condition, there was a systematic increase in pitch strength as the test stimulus varied from WBN to a cosine-phase harmonic tone complex with the reported magnitude judgments being close to 0 for WBN and close to 100 for the cosine-phase harmonic tone complex. In addition, there was a similarity across the four conditions in magnitude judgments for the IIRN stimuli. That is, for IIRN $(-8 \mathrm{~dB})$, the magnitude judgments were around $5-15$ and increased systematically to $50-60$ for IIRN $(-1 \mathrm{~dB})$ across all four conditions. However, there appears to be a difference in magnitude judgements across conditions for the random-phase harmonic tone complex. For the 2-msec condition, the magnitude judgments of pitch strength were similar for the random-phase and cosinephase conditions, with judgments of 96.5 and 98, respectively. As the period of the tone complex increased from 2 to $16 \mathrm{msec}$, there was a greater difference in pitch strength between the random-phase and cosine-phase complex tones. For the 16-msec condition, the magnitude judgments of pitch strength were 55.75 for the random-phase tone complex and 96 for the cosine-phase tone complex.

The individual magnitude judgments from all 15 listeners for the four conditions are shown in Figure 3. Note that there appears to be a similarity in the amount of vari-
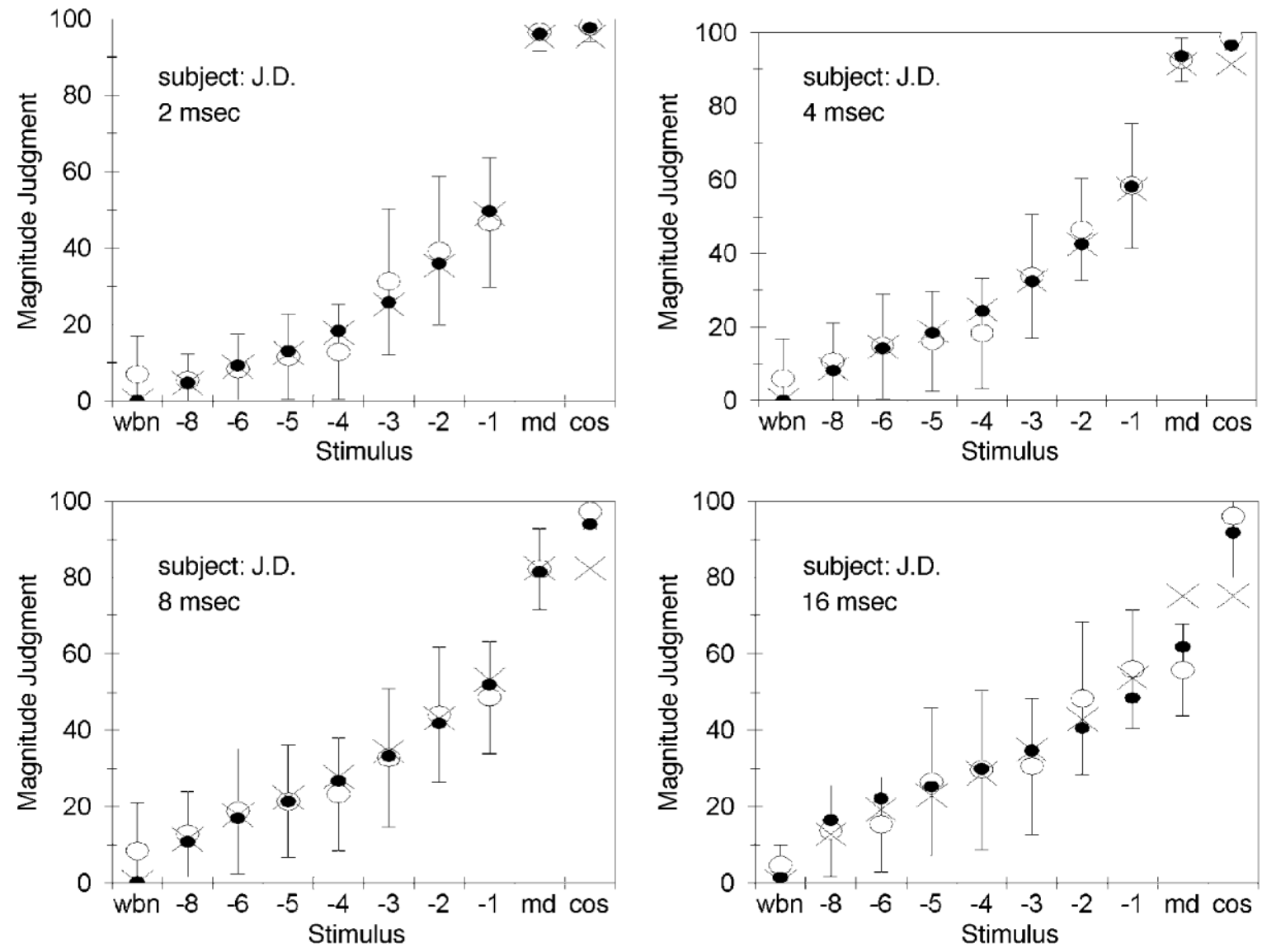

Figure 2. Magnitude judgments of the pitch strengths for the 10 test stimuli for the four different pitch conditions for subject J.D. Open circles and error bars indicate the average magnitude judgments \pm 1 standard deviation. The Xs indicate the predicted magnitude judgments based on Stevens's power law as described by Equation 1. The filled circles indicate the predicted magnitude judgments based on the modification of Stevens's power law as described by Equation 3. 

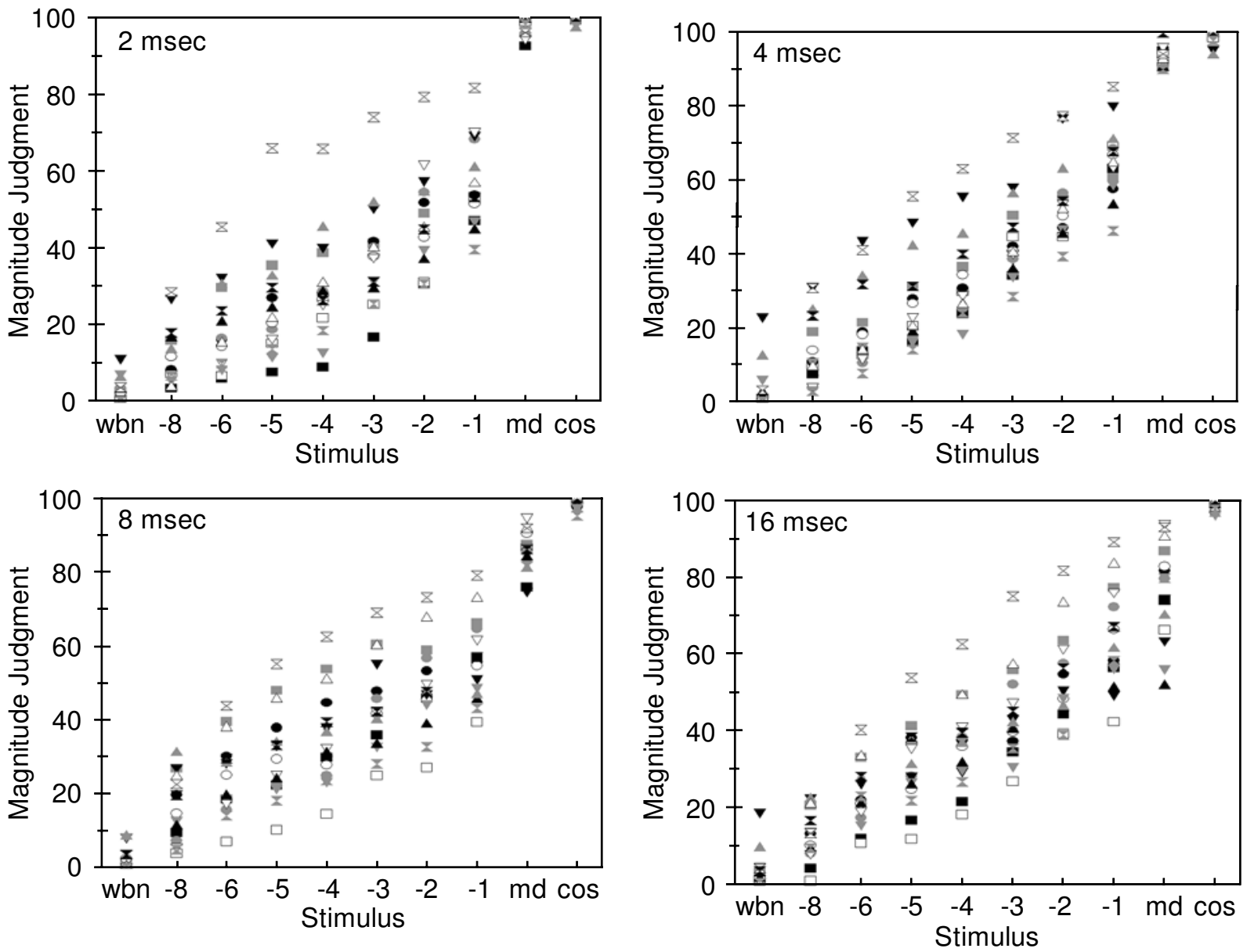

Figure 3. Magnitude judgments of pitch strengths for the 10 test stimuli obtained from 15 listeners for the four different pitch conditions. Symbols indicate different listeners.

ation across conditions for each of the test stimuli, with the exception of the random-phase complex tones. For the random-phase complex tone, there was an increase in the variability across listeners as the period increased from 2 to $16 \mathrm{msec}$. For the 2-msec random-phase condition, all judgments of pitch strength were above 90 , whereas for the 16-msec random-phase condition, judgments of pitch strength varied from 92.6 to 52 . The magnitude judgments of pitch strength for each test stimulus averaged across all 15 listeners for each of the four conditions are compared in Figure 4. Note that across the four conditions, there was a similarity in the average magnitude judgments for each test stimulus, with the exception of the random-phase complex tone. As the period of the random-phase tone complex increased from 2 to $16 \mathrm{msec}$, there was a systematic decrease in the average magnitude judgment of pitch strength from 97.4 to 74.6, respectively (Figure 4).

Figure 5 shows examples of the predicted magnitude judgments of pitch strength based on Stevens's power law (Equation 1) for the test stimuli used in the present study for $\beta=0.5, \beta=1$, and $\beta=2$. Note that the predictions of pitch strength are the same for cosine-phase and random- phase complex tones, because the value of $\phi$ in Equation 1 (i.e., $\mathrm{AC} 1$ of the waveform) was the same for cosine-phase and random-phase tone complexes (see Figure 1). In order to find the best-fitting value for $\beta$ based on empirical data, a log-transform of Stevens's power law is typically applied so that when $\psi$ and $\phi$ are plotted on $\log -\log$ coordinates, the function becomes linear, and the slope of the line is equal to $\beta$ (see Gescheider, 1997). Linear regression was carried out on the function of log-magnitude judgment as a function of the log-waveform $\mathrm{AC} 1$ for the average listener for each condition. In Figure 6, the magnitude judgments averaged across the 15 listeners are compared with the predicted magnitude judgments of pitch strength based on Stevens's power law for each of the 10 test stimuli (Xs in Figure 6). It should be noted that this application of Stevens's power law is a reasonable model of the actual data for most of the test stimuli, but it is not a good model for pitch strengths of the cosine-phase and random-phase complex tones.

The analysis above suggests that there is a small but real contribution of the envelope to pitch strength as the period increases from 2 to $16 \mathrm{msec}$. In order to include a contri- 


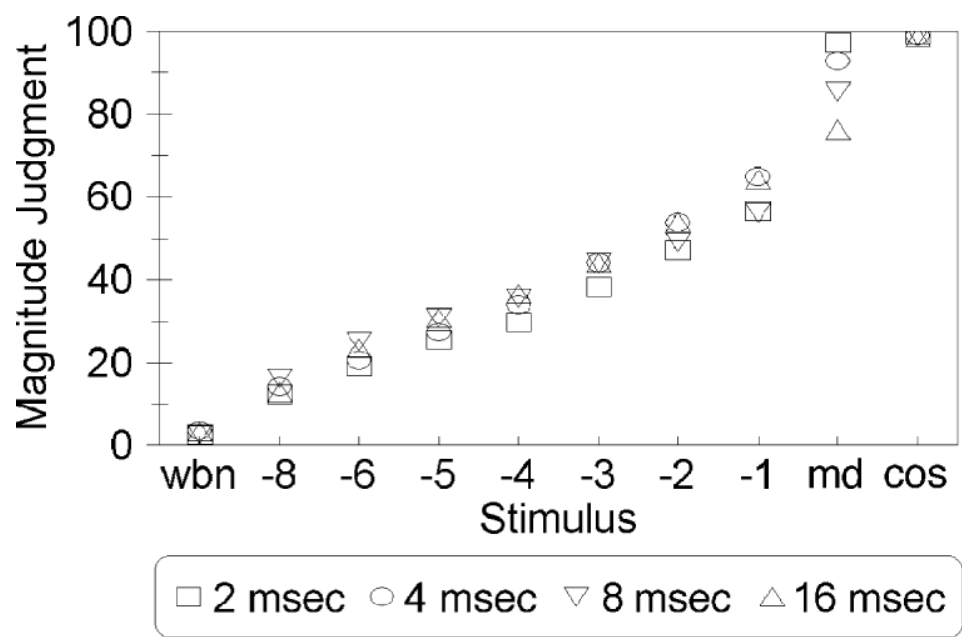

Figure 4. Comparison of the average magnitude judgments of pitch strength from 15 listeners for the four pitch conditions.

bution from the stimulus envelope, Stevens's power law was modified to be

$$
\psi=100\left[k \phi_{\text {wave }}+(1-k) \phi_{\mathrm{env}}\right]^{\beta},
$$

where $\phi_{\text {wave }}$ is $\mathrm{AC} 1$ of the waveform, $\phi_{\mathrm{env}}$ is $\mathrm{AC} 1$ of the envelope, $\beta$ is the exponent, and $k$ is now the relative weight given to the waveform $\mathrm{AC} 1$. Note that appropriate values of $k$ would range between 0 and 1 . The expression within the brackets is multiplied by 100 after exponentiation to express $\psi$ as a number between 0 and 100 . This modification of Stevens's power law implies that pitch strength is determined by a weighted contribution from both the waveform and the envelope. However, because both $\phi_{\text {wave }}$ and $\phi_{\text {env }}$ vary, simple linear regression of the data on $\log -\log$ coordinates cannot be carried out in order to estimate $\beta$. Consequently, the data were fit to the modification above of Stevens's power law by using an iterative algorithm in order to find the best-fitting values of $\beta$ and $k$. The filled circles in Figure 6 show the predicted magnitude judgments for each of the 10 test stimuli based on this modification to Stevens's power law for the average listener; the best-fitting values of $\beta$ are 2.02 , $1.76,1.48$, and 1.34 for the $2-, 4-, 8-$, and 16 -msec conditions, respectively, and the best-fitting values of $k$ are 0.98 , $0.97,0.89$, and 0.86 for the $2-, 4-, 8-$, and $16-m s e c$ conditions, respectively. Comparison of the filled circles with the

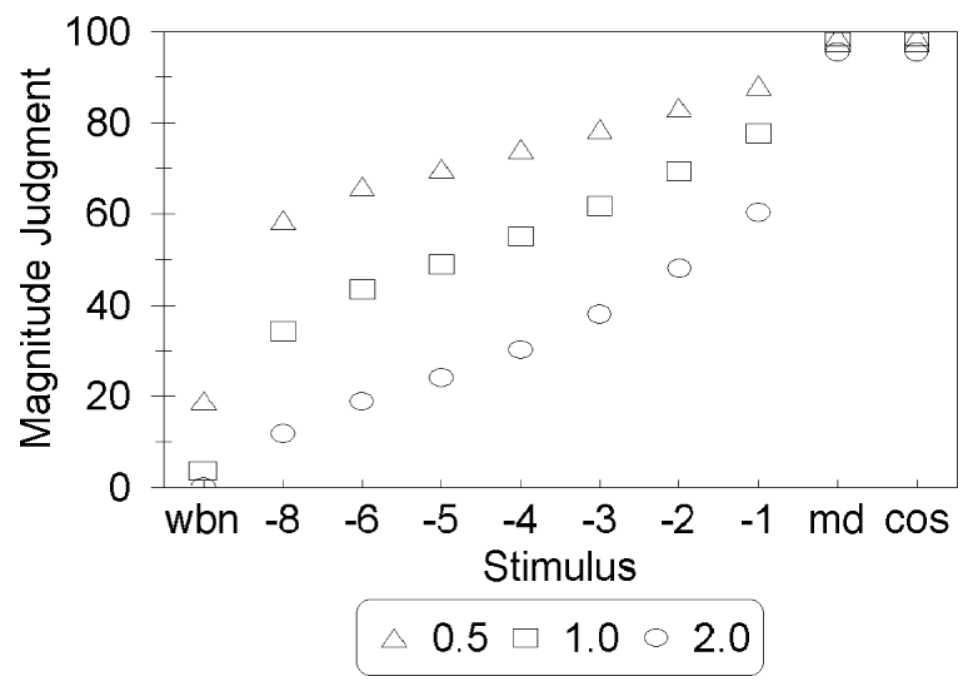

Figure 5. Predicted magnitude judgments for the 10 test stimuli based on Stevens's power law (Equation 1) for values of $\beta$ equal to 0.5 , 1, and 2 . Values of $\beta$ were chosen to illustrate the predictions when $\beta<1, \beta=1$, and $\beta>1$. Note that regardless of the value of $\beta$, the predicted magnitude judgments were equal for cosine-phase (cos) and random-phase (rnd) complex tones. 
Xs in Figure 6 suggests that the modification of Stevens's power law based on both the waveform and the envelope is a better predictor of the pitch strengths of cosine-phase and random-phase complex tones, although the inclusion of the envelope AC1 does not appear to substantially improve the predictions of pitch strength for the IIRN stimuli. The residual sum of squares between the average data and the predictions of Stevens's power law are larger than the residual sum of squares between the average data and the predictions of the modified Stevens's power law for each condition (Table 1). In general, this modification of Stevens's power law based on both waveform and envelope autocorrelation functions is a good predictor of pitch strength for all of the stimuli tested, including the relative pitch strengths of the cosine-phase and random-phase complex tones.

The magnitude judgments from each of the individual listeners were also fit to the modification above of Stevens's power law by using an iterative algorithm in order to find the best-fitting values of $\beta$ and $k$. For listener J.D. (see Fig- ure 2), the best-fitting values of $\beta$ were $2.89,2.35,1.92$, and 1.18 for the 2-, 4-, 8-, and 16-msec conditions, respectively, and the best-fitting values of $k$ were $0.99,0.98,0.9$, and 0.62 for the 2-, 4-, 8-, and 16-msec conditions, respectively. Comparison of the filled circles to the Xs in Figure 2 again suggests that the modification of Stevens's power law based on both the waveform and the envelope is a better predictor of the pitch strengths of cosine-phase and random-phase complex tones, although the inclusion of the envelope AC1 does not appear to substantially improve the predictions of pitch strength for the IIRN stimuli. Values of the best-fitting $\beta$ and $k$ are shown in Table 2 and Table 3 , respectively, for individual listeners, with the averaged values shown in Figure 7. The ranges of individual best-fitting $\beta$ s were $0.84-3.47$ at 2 msec, $0.85-2.63$ at $4 \mathrm{msec}, 0.95-3.08$ at $8 \mathrm{msec}$, and $0.38-2.05$ at $16 \mathrm{msec}$. Although listeners J.B.K. and R.S. have $\beta$ s consistently less than 1 , the other 13 listeners consistently had $\beta$ s greater than or equal to 1 (Table 2 ; see also note 1 ). There was a systematic decrease in the average $\beta$ as the period or
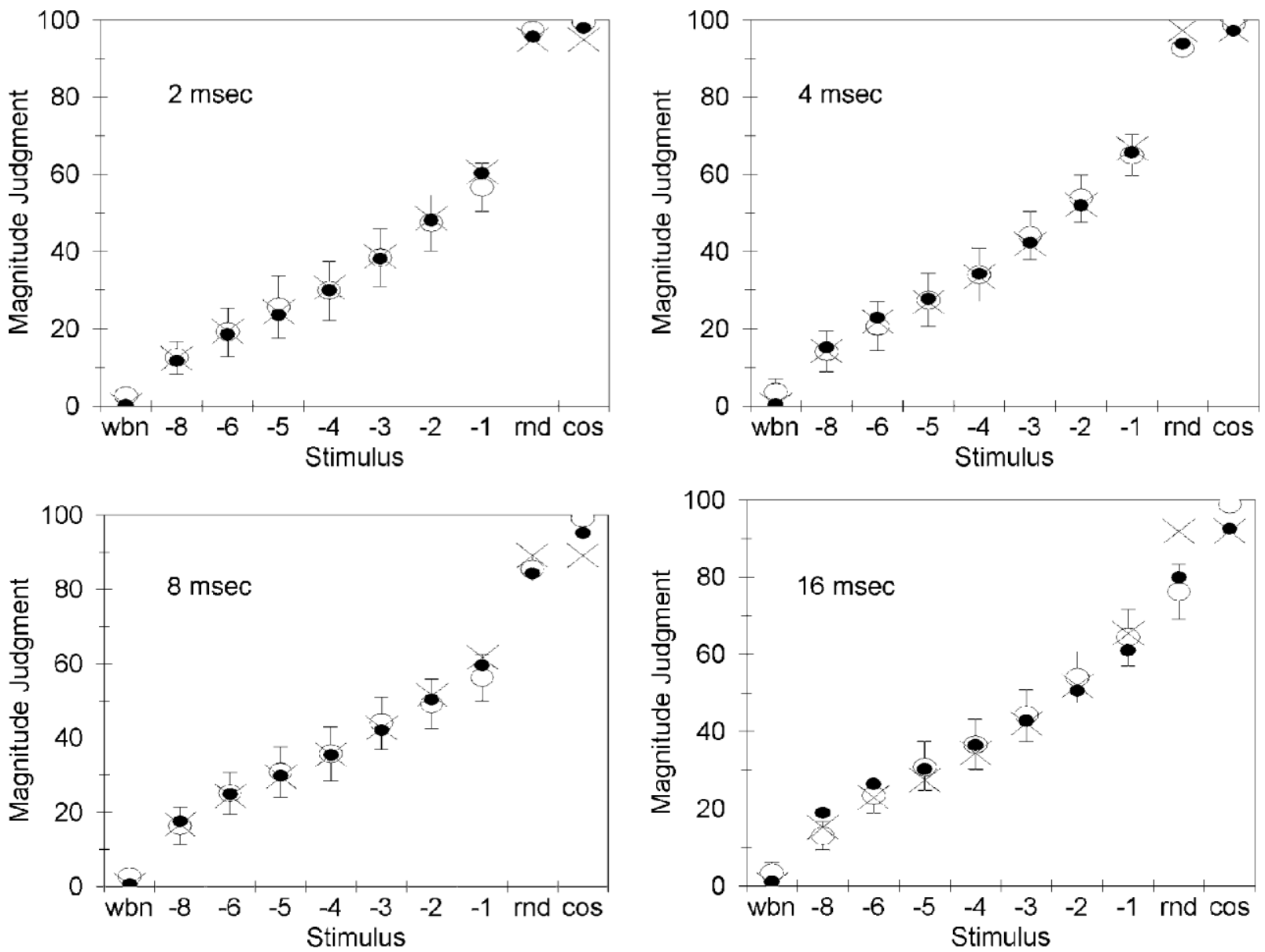

Figure 6. Average magnitude judgments of the pitch strengths for the 10 test stimuli for the four different pitch conditions from 15 listeners. Open circles and error bars indicate the average magnitude judgments \pm the $95 \%$ confidence intervals. The Xs indicate the predicted magnitude judgments based on Steven's power law as described by Equation 1. The filled circles indicate the predicted magnitude judgments based on the modification of Stevens's power law as described by Equation 3. 
Table 1

Residual Sum of Squares Between the Average Magnitude Judgments and the Predictions Based on Stevens's power law and the Exponential Function Described by Yost (1996b)

\begin{tabular}{lrrrr}
\hline & \multicolumn{4}{c}{ Condition } \\
\cline { 2 - 5 } \multicolumn{1}{c}{ Model } & $2 \mathrm{msec}$ & $4 \mathrm{msec}$ & $8 \mathrm{msec}$ & $16 \mathrm{msec}$ \\
\hline Steven's power law (Equation 1) & 52.52 & 47.46 & 157.16 & 332.58 \\
Modified power law (Equation 3) & 30.52 & 28.42 & 39.54 & 129.20 \\
Exponential Function (Equation 2; $a=10, b=2)$ & 616.57 & $1,192.67$ & $1,255.82$ & $1,840.30$ \\
Exponential Function (Equation 2; best $a$ and $b$ ) & 431.08 & 767.44 & 705.64 & $1,159.20$ \\
Best value of $a$ for Equation 2 & 14.40 & 18.30 & 19.40 & 20.10 \\
Best value of $b$ for Equation 2 & 1.95 & 1.93 & 1.92 & 1.95 \\
\hline
\end{tabular}

delay increased from 2 to $16 \mathrm{msec}$; a repeated measures analysis of variance (ANOVA) showed that there was a significant difference across conditions $(F=18.045 ; F>$ $\left.F_{0.0005(1), 3,42}\right)$. The ranges of individual best-fitting $k \mathrm{~s}$ were $0.83-1.0$ at $2 \mathrm{msec}, 0.89-1.0$ at $4 \mathrm{msec}, 0.65-1.0$ at $8 \mathrm{msec}$, and $0-1$ at $16 \mathrm{msec}$. A repeated measures ANOVA showed that there was a significant difference across conditions for the average values of $k(F=6.275$; $\left.F>F_{0.0025(1), 3,42}\right)$.

\section{DISCUSSION}

Fastl and Stoll (1979) used a magnitude estimation procedure to scale the strength of pitch for a variety of different types of stimuli that produced pitches matched to 125 , 250 , and $500 \mathrm{~Hz}$. The periods of these stimuli were 8, 4, and $2 \mathrm{msec}$, respectively. In general, listeners rated stimuli that can be characterized by line spectra as having greater pitch strength than stimuli characterized by continuous spectra. For the stimuli used in the present experiment, harmonic tone complexes were characterized by line spectra, whereas IIRNs were characterized by continuous spectra. However, the range of frequencies comprising each test stimulus varied across stimuli in the Fastl and Stoll study, and consequently, there were potential variations in timbre, in addition to the variations in pitch strength. Singh and Hirsh (1992) have shown that timbre is primarily affected by changes in the spectral location of components, whereas pitch is primarily affected by changes in $f 0$. In the present study, the same range of frequencies was used for all test stimuli (i.e., frequencies up to and including $10 \mathrm{kHz}$ ). Consequently, there should be systematic variations in pitch strength across our stimuli with minimal timbre changes. ${ }^{3}$ In agreement with the results of Fastl and Stoll, the results of the present study show that the perceived pitch strengths of stimuli characterized by line spectra (i.e., harmonic tone complexes) were larger than those of stimuli characterized by continuous spectra (i.e., IIRNs).

It has been argued that pitch strength is directly related to the height of $\mathrm{AC} 1$ in the stimulus autocorrelation function (Patterson et al., 1996; Yost, 1996b; Yost, Patterson, $\&$ Sheft, 1996). With the use of a scaling procedure to measure the pitch strengths of iterated rippled noises, Yost (1996b) described the relationship between AC1 and pitch strength with the exponential function described by
Equation 2. Neither Fastl and Stoll (1979) nor Yost (1996b) evaluated pitch strength in terms of Stevens's power law as described by Equation 1. The results of the present study show that the pitch strength can be related to AC1 with the use of Stevens's power law. Magnitude judgments of pitch strengths of IIRNs can be predicted by Stevens's power law by using Equation 1 as indicated in Figures 2 and 6 . However, Stevens's power law predicts that the pitch strengths should be equal for cosine-phase and random-phase harmonic complex tones, because the $\mathrm{AC} 1 \mathrm{~s}$ of the waveforms are identical for cosine-phase and random-phase conditions (see Figure 1). In contrast, the listeners' magnitude judgments of the pitch strengths for random-phase complex tones were generally smaller than those for cosinephase complex tones, particularly as the period of the stimulus increased from 2 to $16 \mathrm{msec}$. Also note that, although the height of $\mathrm{AC} 1$ did vary systematically over a range of $0-1$, many of the pitch strength judgments were between 10-60 and that an equal number of judgments did not occur above 60 . Of the 10 stimuli tested, 5 had values of $\mathrm{AC} 1$ greater than 0.6, and 5 had values of $\mathrm{AC} 1$ below 0.6. The fact that the perceived pitch strengths were not judged to be equally spaced above and below 60 presumably reflects the nonlinearnature of the pitch strength function. A similar observation is noted in Yost (1996b).

Table 2

Values of the Best-Fitting Exponent $(\beta)$ in the Modification of Stevens's power law for Individual Listeners

\begin{tabular}{lcccc}
\hline & \multicolumn{4}{c}{ Condition } \\
\cline { 2 - 5 } Subject & $2 \mathrm{msec}$ & $4 \mathrm{msec}$ & $8 \mathrm{msec}$ & $16 \mathrm{msec}$ \\
\hline A.M. & 3.47 & 2.38 & 1.67 & 1.94 \\
D.G. & 1.67 & 1.53 & 0.95 & 1.16 \\
E.A. & 2.21 & 2.10 & 3.08 & 2.05 \\
E.O. & 1.96 & 1.87 & 1.22 & 1.39 \\
G.S. & 2.02 & 2.18 & 1.84 & 1.49 \\
H.H. & 2.34 & 1.80 & 1.73 & 1.66 \\
J.B.K. & 1.38 & 0.85 & 0.95 & 0.38 \\
J.D. & 2.89 & 2.35 & 1.92 & 1.18 \\
J.V. & 2.11 & 2.15 & 1.96 & 1.51 \\
R.D. & 2.31 & 2.19 & 1.83 & 0.97 \\
R.K. & 1.48 & 1.13 & 1.04 & 1.02 \\
R.N. & 2.20 & 2.07 & 1.09 & 1.14 \\
R.S. & 0.84 & 0.91 & 0.95 & 0.90 \\
S.K. & 2.06 & 1.55 & 1.36 & 1.13 \\
W.M. & 3.14 & 2.63 & 2.29 & 1.76 \\
\hline
\end{tabular}


Table 3

Values of the Best-Fitting Weight for the Waveform $(k)$ in the Modification of Stevens's power law for Individual Listeners

\begin{tabular}{lcccc}
\hline & \multicolumn{4}{c}{ Condition } \\
\cline { 2 - 5 } Subject & $2 \mathrm{msec}$ & $4 \mathrm{msec}$ & $8 \mathrm{msec}$ & $16 \mathrm{msec}$ \\
\hline A.M. & 0.99 & 1.00 & 0.86 & 0.90 \\
D.G. & 0.96 & 0.93 & 0.86 & 0.97 \\
E.A. & 0.83 & 1.00 & 0.97 & 0.83 \\
E.O. & 0.96 & 0.95 & 0.85 & 0.88 \\
G.S. & 1.00 & 1.00 & 0.97 & 0.95 \\
H.H. & 1.00 & 0.96 & 0.94 & 0.95 \\
J.B.K. & 0.98 & 0.89 & 0.65 & 0.00 \\
J.D. & 0.99 & 0.98 & 0.90 & 0.62 \\
J.V. & 1.00 & 1.00 & 1.00 & 1.00 \\
R.D. & 0.98 & 0.96 & 0.89 & 0.50 \\
R.K. & 0.96 & 0.91 & 0.69 & 0.68 \\
R.N. & 1.00 & 0.99 & 0.97 & 1.00 \\
R.S. & 1.00 & 1.00 & 1.00 & 1.00 \\
S.K. & 0.98 & 0.99 & 0.87 & 0.81 \\
W.M. & 0.99 & 0.96 & 0.91 & 0.90 \\
\hline
\end{tabular}

Although the waveform $\mathrm{AC} 1 \mathrm{~s}$ are identical for cosinephase and random-phase tone complexes, the $\mathrm{AC} 1 \mathrm{~s}$ of the stimulus envelopes did differ between phase conditions. The envelope of the cosine-phase complex tone was highly modulated (i.e., "peaky") compared with the relatively "flat" envelope of the corresponding random-phase condition. Consequently, the envelope $\mathrm{AC} 1$ was large for cosinephase stimuli and small for random-phase stimuli. The magnitude judgments given by the listeners for cosinephase and random-phase complex tones suggests that the stimulus envelope had some influence on the perceived pitch strength. In order to account for the influence of the stimulus envelope, a modification of Stevens's power law as described by Equation 3 was used. This modification of Stevens's power law implies that pitch strength is based on a weighted average of the temporal information in both the waveform and the envelope. This modified version of the power law can accurately predict the magnitude judgments for cosine-phase complex tones, random-phase complex tones, and IIRNs. Note that, although the unmodified power law in Equation 1 can be used to predict the pitch strength for most stimuli, it does not account for the pitch strengths of stimuli at the extremes of the pitch strength scale where pitch strength is the largest (Figure 8). That is, Stevens's power law deviates from the perceived pitch strengths of stimuli that actually produce the most salient pitches. In this regard, it is interesting to note that modifications to Stevens's power law have been implemented in order to account for the deviations in the magnitude of sensations near absolute threshold (i.e., at the other extreme of the perceptual scale; see Marks and Stevens, 1968).

Based on the general shape of the exponential function (Equation 2) described by Yost (1996b), it would be predicted that the exponent, $\beta$, in Stevens's power law for pitch strength would be greater than 1 . The results of the present study show that the value of the exponent in the modification of Stevens's power law is indeed greater than 1, indicating that the growth of pitch strength increases at a faster rate of change does than the height of AC1. This is in contrast to loudness, which has an exponent of less than 1 (e.g., Hellman, 1976, 1981; Logue, 1976; J. C. Stevens \& Guirao, 1964; S. S. Stevens, 1955, 1975) and which increases at a slower rate of change than does the sound pressure level. That is, the perception of pitch strength is expansive, whereas the perception of loudness is compressive. The average value of the exponent decreased systematically as the period of the stimulus increased from 2 to 16 msec (i.e., $\beta$ decreased as the pitch decreased from 500 to $62.5 \mathrm{~Hz}$; see top panel of Figure 7). The systematic decrease in the value of the exponent is primarily due to the weaker pitch strength assigned to the random-phase tone complexes as the period increased from 2 to $16 \mathrm{msec}$ (Figure 4), whereas the magnitude judgments for the IIRN stimuli remained about the same across all four conditions (Figure 4).

For comparison with the power law, the present magnitude judgments were also fit with the exponential function (Equation 2) as described by Yost (1996b). The exponential function does not predict magnitude judgments as well as the power function does (Figure 8). Table 1 summarizes the residual sum of squares for each model and shows that the best fits are obtained when the modified version of Stevens's power law is used. Although the power law and the exponential function are both expansive, the exponential function increases at a faster rate of change than do the actual magnitude judgments (Figure 8), primarily because the value of $\mathrm{AC} 1$ is an exponential term rather than a multiplicative term, as in the power law. It should be noted that the exponential function (Equation 2) where $a=10$ and $b=2$ as described by Yost (1996b) can be approximated with a power function having the form

$$
\psi=11.6+\left(100 * \mathrm{AC}^{4.0}\right) .
$$

It is unclear why the exponential model can account for the data of Yost (1996b), but did not predict our results. One difference between the studies is the stimulus that was assigned the maximum pitch strength. In the present study, maximum pitch strength was assigned to the cosine-phase harmonic tone complex, whereas Yost (1996b) assigned maximum pitch strength to an iterated rippled noise of 256 iterations.

Yost, Patterson, and Sheft (1998) have shown that the temporal information in the stimulus envelope cannot account for pitch discrimination, but that the envelope does contain some information for the discrimination of iterated rippled noise from WBN. These authors concluded that pitch is primarily based on the temporal information in the waveform fine structure. The results of the present study indicate that the temporal information in the stimulus envelope, as measured by the height of $\mathrm{AC} 1$, can also provide a small contribution to the perception of pitch strength. The average values of the weight $(k)$ given to the waveform AC1 ranged from 0.97 at 2 and $4 \mathrm{msec}$ to 0.8 at $16 \mathrm{msec}$ obtained from individual data, and ranged from 0.98 at $2 \mathrm{msec}$ to 0.86 at $16 \mathrm{msec}$ based on the fits to the average 

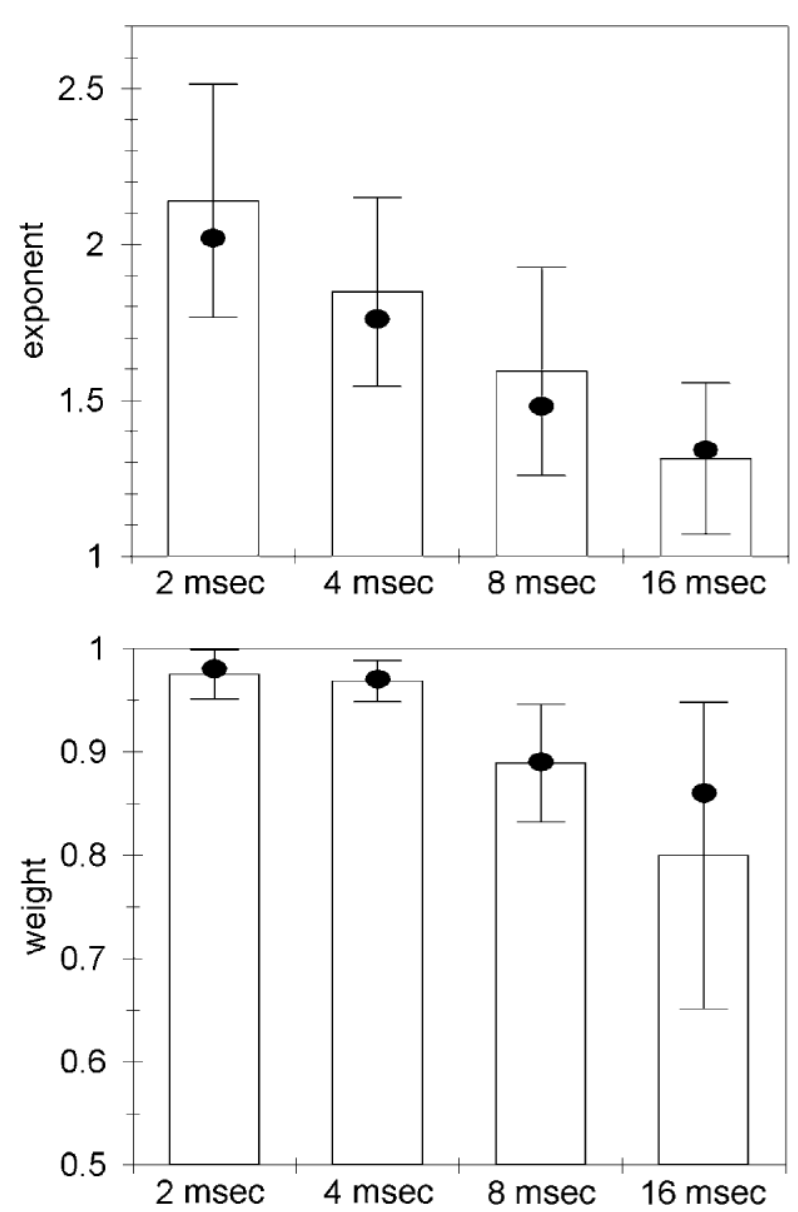

Figure 7. The top panel shows the histogram of the average values of the exponent $(\beta)$ obtained from individual best fits to the modification of Stevens's power law (Equation 3) for the four pitch conditions from 15 listeners. The bottom panel shows the histogram of the average values of the weight $(k)$ obtained from individual best fits to the modification of Stevens's power law (Equation 3 ) for the four pitch conditions from 15 listeners. Error bars indicate the $95 \%$ confidence intervals. The filled circles indicate the best fitting $\beta$ and $k$ based on the magnitude judgments averaged across the 15 listeners (see Figure 6).

data (see bottom panel of Figure 7). These results suggest that, although the envelope can influence saliency, pitch strength is primarily determined by the fine structure of the stimulus. That is, listeners presumably combine temporal information across all frequency channels, but rely most heavily on information in low-frequency channels where information about the fine structure is dominant. Although the contribution to pitch strength is small from those channels that can only preserve temporal information about the envelope, the envelope contribution increases in weight as the period increases (see bottom panel of Figure 7).

The temporal information that is available within the auditory system about the fine structure and the envelope of the stimulus can be illustrated with the auditory image model (AIM) of Patterson, Allerhand, and Giguère (1995).
Figure 9 illustrates the outputs of the four stages of AIM for the 8-msec cosine-phase harmonic tone complex for 32 auditory filters centered between 100 and $10000 \mathrm{~Hz}$. The frequency analysis that is carried out by the basilar membrane is simulated by a bank of gamma-tone filters. This is the spectral analysis stage of AIM and shows the output of each auditory filter as a function of time. The transduction process is simulated by the neural activity pattern, which is accomplished by half-wave rectification, compression, and lowpass filtering of the output of the gamma-tone filter bank. This is the neural encoding stage of AIM and represents the average pattern of activity in the auditory nerve. Autocorrelation of each frequency channel in the neural activity pattern is carried out to form an auditory image known as the autocorrelogram. This is the temporal integration stage and produces a stable, visual image of the sound that is presumably related to the auditory perception of the sound. The temporal information in the autocorrelogram can be summarized by adding across all of the frequency channels to yield the summary autocorrelogram. For the 8-msec cosine-phase harmonic tone complex, there is a large peak at $0 \mathrm{msec}$ followed by peaks at $8 \mathrm{msec}$ and integer multiples of $8 \mathrm{msec}$ in the summary autocorrelogram (see bottom right-hand panel in Figure 9); these peaks represent the temporal information within the auditory nervous system and are analogous to the peaks in the stimulus autocorrelation functions shown in Figure 1.

It is known that auditory nerve fibers show strong phaselocking to frequencies at or below $1000 \mathrm{~Hz}$ and that the strength of phase-locking decreases as frequency increases so that above $3000 \mathrm{~Hz}$, phase-locking no longer exists (Johnson, 1980; Palmer \& Russell, 1986). Consequently, auditory nerve fibers having best frequencies below $1000 \mathrm{~Hz}$ show strong phase-locking to the waveform fine structure, whereas auditory nerve fibers having best frequencies above $3000 \mathrm{~Hz}$ can only show phase-locking to the low frequencies that can be found in the stimulus envelope (e.g., Joris \& Yin, 1992). This phenomenon is captured by AIM and can be seen in the neural activity pattern and autocorrelogram in Figure 9. Auditory channels at or below $1000 \mathrm{~Hz}$ are dominated by the temporal properties of the fine structure, whereas auditory channels at or above $3000 \mathrm{~Hz}$ are dominated by the temporal properties of the envelope.

In Figure 10, the summary autocorrelograms obtained from 25 channels between $100-1000 \mathrm{~Hz}$ are compared with the summary autocorrelograms obtained from 22 channels between $3000-10000 \mathrm{~Hz}$ for cosine-phase and random-phase harmonic complex tones across the four conditions in the present study. ${ }^{4}$ These summary autocorrelograms represent the information available within the auditory system for each stimulus condition on the basis of the fine structure alone (i.e., channels 100$1000 \mathrm{~Hz}$ ) and the envelope alone (i.e., channels 3000$10000 \mathrm{~Hz}$ ). For frequency channels dominated by the fine structure (thin lines in Figure 10), there is virtually no difference in the relative heights of the peaks between 

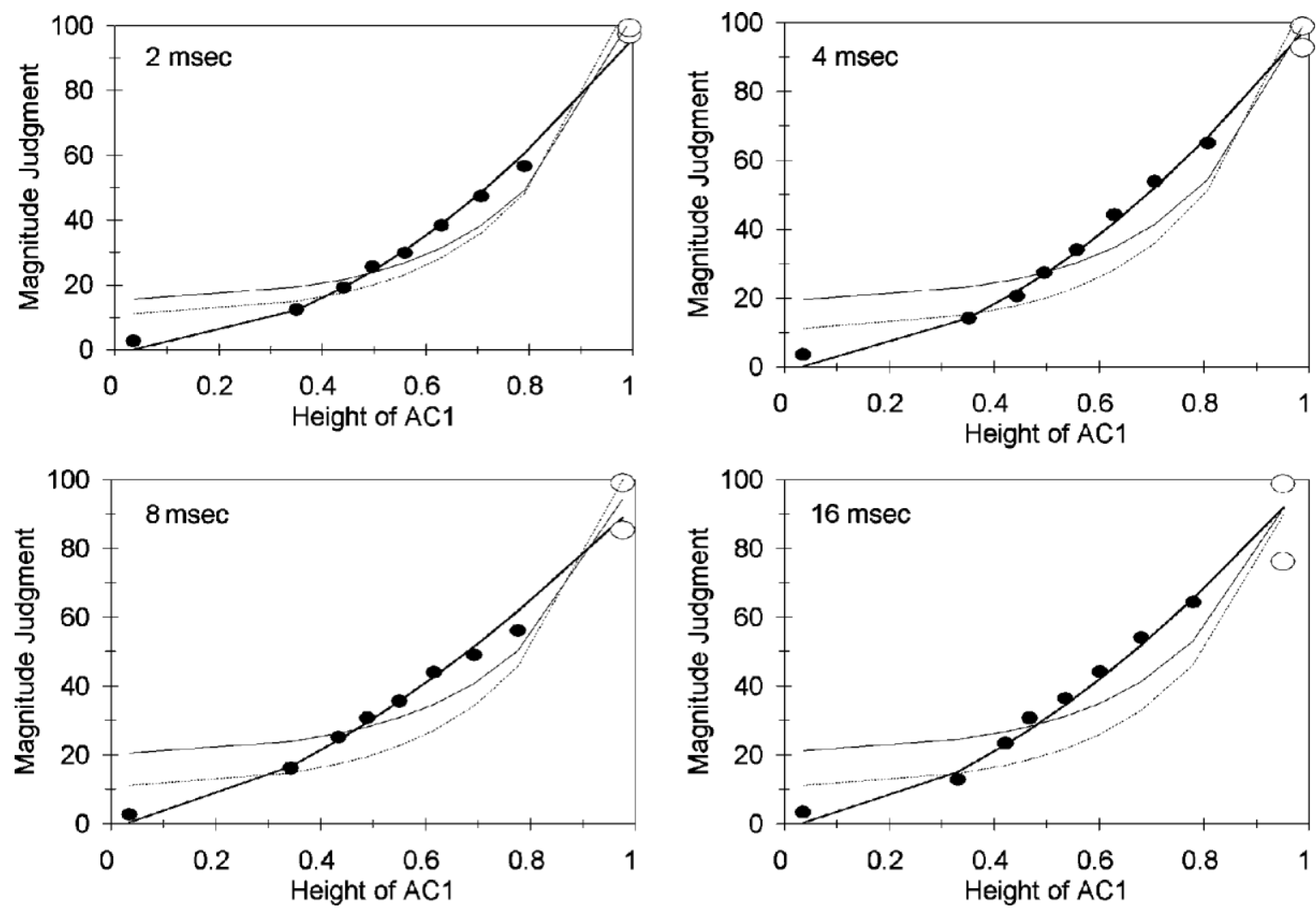

Figure 8. Magnitude judgments averaged across 15 listeners as a function of the height of AC1 for the four pitch conditions. Average magnitude judgments for WBN and IIRN are indicated by the small, filled circles. The random-phase and cosine-phase harmonic complex tones are indicated by the large, open circles. The thick solid lines indicate the predictions based on Stevens's power law as described by Equation 1. Note the systematic deviation that occurred between the predictions of Stevens's power law and the magnitude judgments for those stimuli giving the most salient pitches (open circles). The dotted lines show the predictions of the exponential function (Equation 2) where $a=10$ and $b=2$ as described by Yost (1996b). The thin solid lines show the predictions of the exponential function where the best-fitting values of $a$ and $b$ (see Table 1) were determined using an iterative procedure.

the summary autocorrelograms for cosine-phase stimuli and the corresponding summary autocorrelograms for the random-phase stimuli in all four conditions. In each summary autocorrelogram, there are large, clear peaks at the appropriate time lags.

In contrast, the summary autocorrelograms based on the frequency channels dominated by the envelope (thick lines in Figure 10) show two different effects. First, for the cosinephase autocorrelograms, there is an increase in the relative peak heights as the period increases from 2 to $16 \mathrm{msec}$. That is, there is an enhancement in the strength of the envelope information for large periods (i.e., low pitches) compared with the envelope information available for small periods (i.e., high pitches). For the 8 - and 16-msec periods, the relative peak heights in the envelope autocorrelograms are actually larger than the relative peak heights of the fine structure autocorrelograms (see the left-hand column of Figure 10). This suggests that the temporal information within the auditory system regarding the stimulus envelope is better for large periods (i.e., low pitches) than for small periods (i.e., high pitches) and is consistent with the finding that the relative weight given to the envelope (i.e., $1-k$ ) increases as period increases (i.e., bottom panel of Figure 7). Second, there is a clear difference in the quality of the envelope information between cosine-phase and random-phase autocorrelograms. That is, the envelope peak heights are smaller for the random-phase autocorrelograms compared with the envelope peak heights in the cosine-phase autocorrelograms for each of the four conditions (i.e., compare the thick lines in the right-hand column with those in the left-hand column of Figure 10). Also note that the envelope peak heights in the cosinephase autocorrelograms are smaller for the 2-msec condition than for the 16-msec condition, whereas the opposite is true for the random-phase conditions. That is, the envelope peak heights for the 2-msec random-phase autocorrelogram appear to be larger than those for the corresponding 16-msec condition. Thus, the relative difference in the envelope peak heights between cosine-phase and randomphase autocorrelograms becomes more obvious as the period of the stimulus increases from 2 to $16 \mathrm{msec}$ (i.e., as the pitch decreases). 


\section{Gamma-Tone Filter Bank}
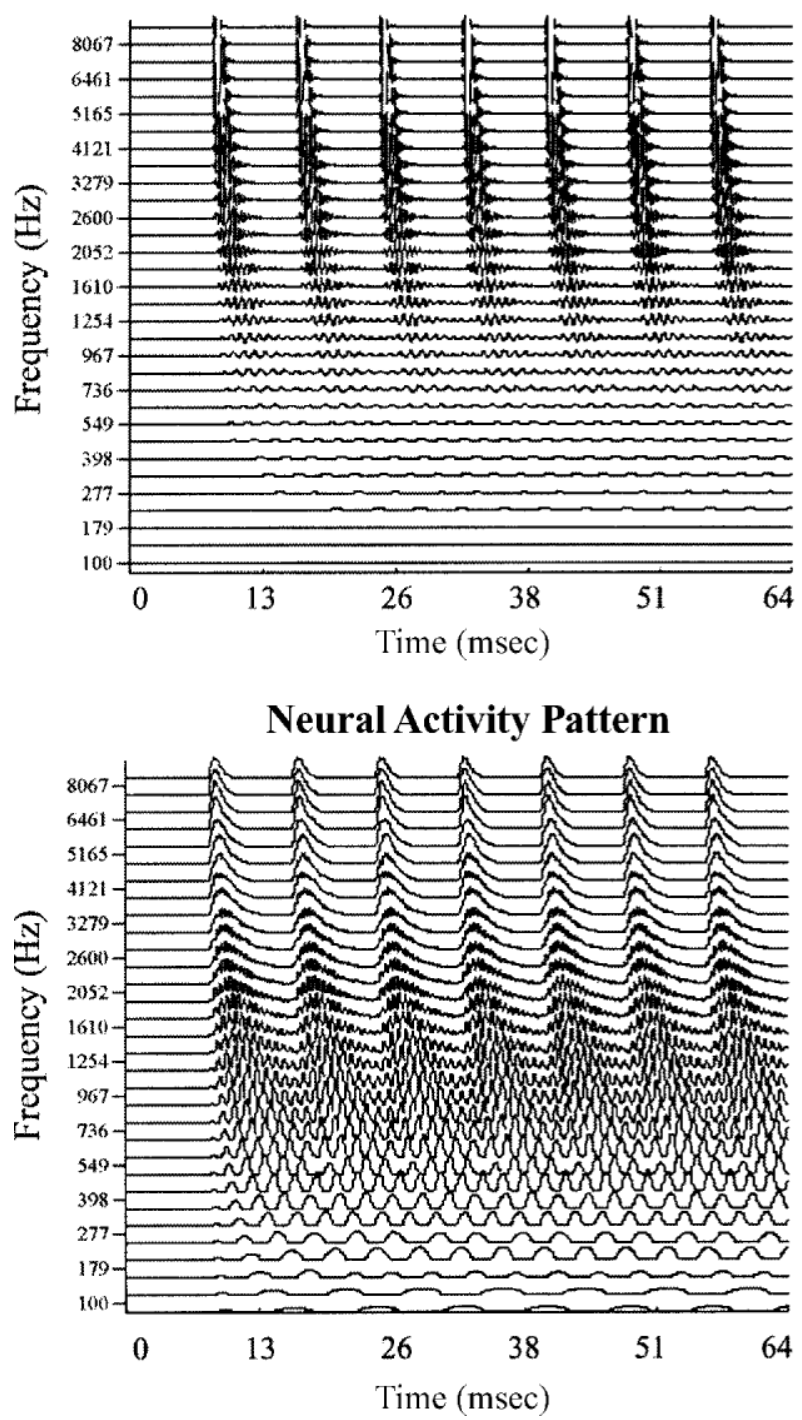

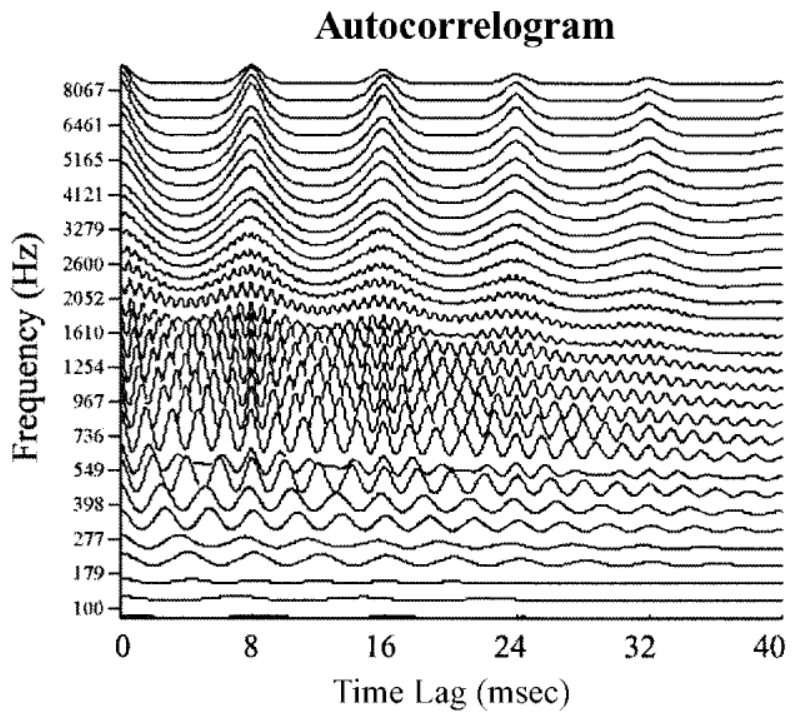

Summary Autocorrelogram

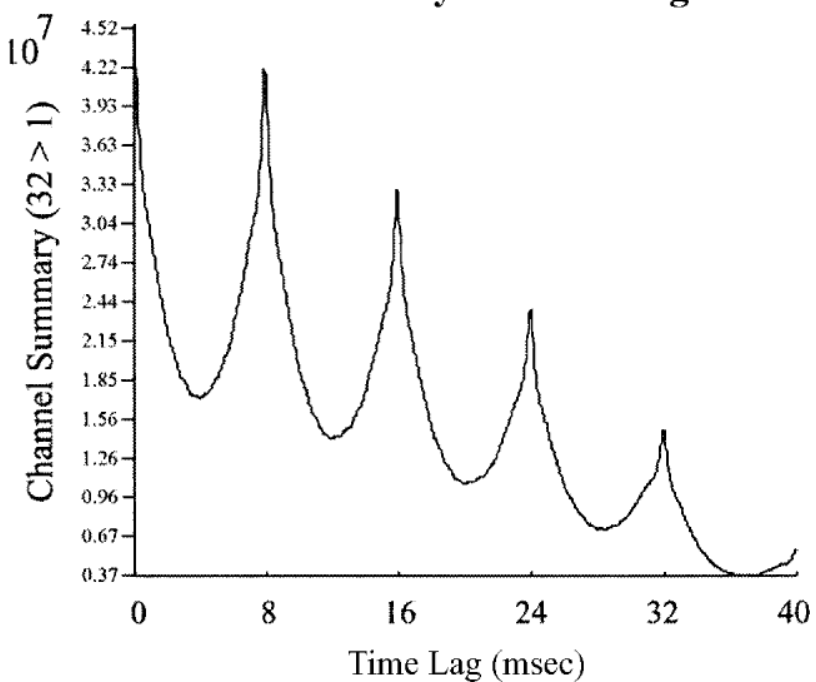

Figure 9. Outputs of the auditory image model to a cosine-phase harmonic tone complex having a period of $8 \mathrm{msec}$. The four stages of the model simulate basilar membrane filtering (gamma-tone filter bank), the pattern of activity in the auditory nerve (neural activity pattern), and tem poral integration of the auditory nerve activity (autocorrelogram). The temporal information can be summarized by adding the autocorrelograms across auditory filters (summary autocorrelogram).

As previously mentioned, listeners presumably combine temporal information across all frequency channels, but rely most heavily on information in low-frequency channels where information about the fine structure is dominant. With respect to the summary autocorrelograms in Figure 10, this implies that pitch strengths for the cosinephase and random-phase harmonic tone complexes will be determined by the weighted average of the fine structure (shown by the thin lines) and the envelope autocorrelograms (shown by thick lines). Figure 10 allows us to make two predictions based on AIM in regard to pitch strength. First, for any given period (i.e., pitch), it should be clear that even if the relative contribution of the envelope is small (i.e., channels 3000-10000 Hz), there will be some decrease in the relative pitch strength of a random-phase tone complex compared with that of the cosine-phase tone complex. This prediction is consistent with the magnitude judgments of the listeners in the present study. Second, as the period increases from 2 to $16 \mathrm{msec}$, the pitch strengths of the random-phase complexes relative to the cosinephase tone complexes should become weaker. That is, the envelope will have a larger effect on pitch strength at $16 \mathrm{msec}$ than at $2 \mathrm{msec}$. This prediction is consistent with the finding that the relative weight given to the envelope in the modified power law (i.e., $1-k$ ) increases as the period increases. 

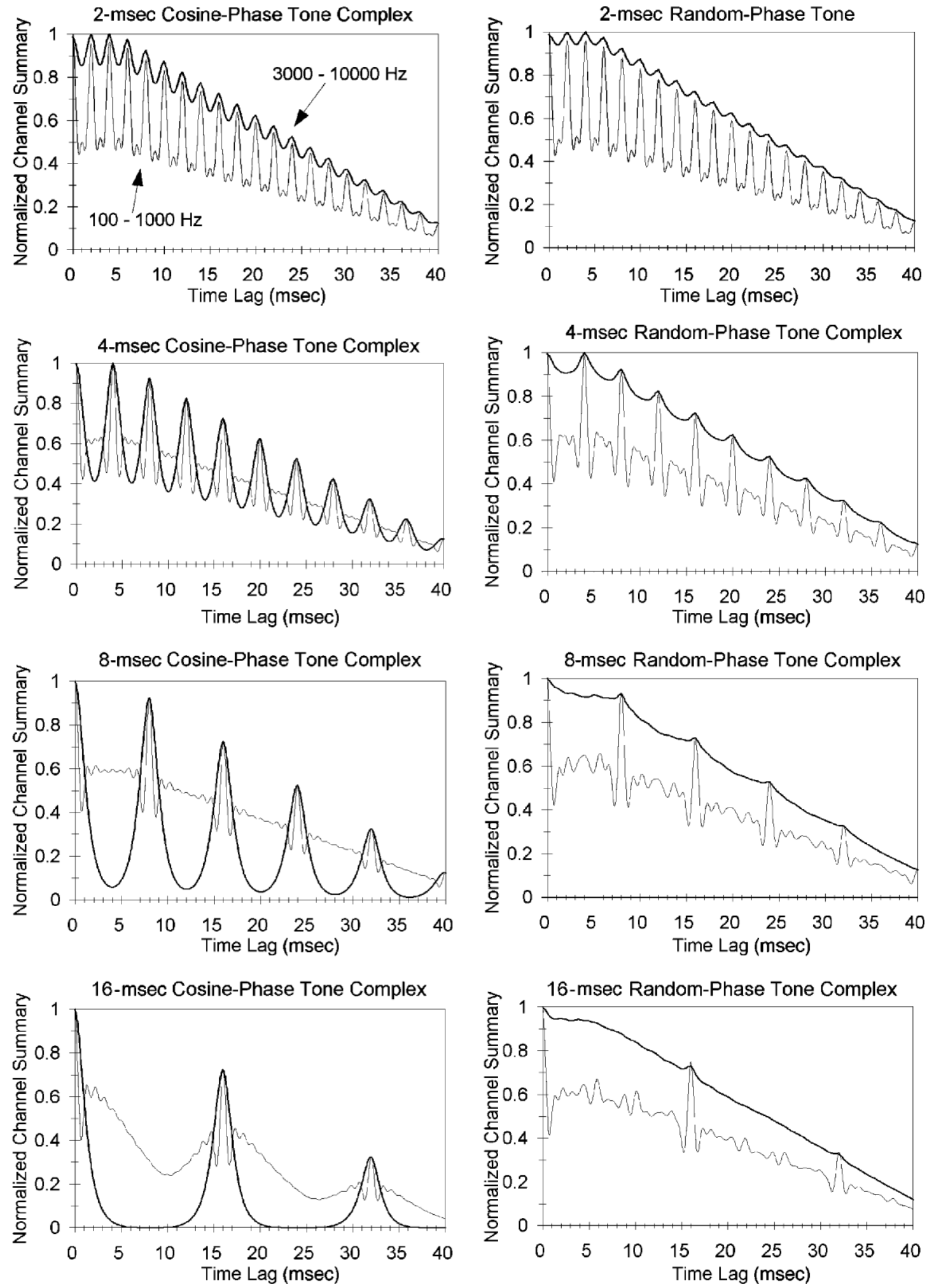

Figure 10. Summary autocorrelograms from the auditory image model (AIM) for the cosine-phase (left-hand column) and randomphase (right-hand column) harmonic tone complexes in the present study. Thin lines show summary autocorrelograms obtained from frequency channels between $100-1000 \mathrm{~Hz}$, and thick lines show summary autocorrelograms obtained from frequency channels between $3000-10000 \mathrm{~Hz}$, as indicated by the arrows. Each summary autocorrelogram has been normalized to the value at a time lag of 0 msec in a manner similar to that of the stimulus autocorrelation functions in Figure 1. 


\section{REFERENCES}

Fastl, H., \& Stoll, G. (1979). Scaling of pitch strength. Hearing Research, 1, 293-301.

GESCHEIDER, G. A. (1997). Psychophysics: The fundamentals(3rd ed.). Mahwah, NJ: Erlbaum.

Hellman, R. P. (1976). Growth of loudness at 100 and 3000 Hz. Journal of the Acoustical Society of America, 60, 672-679.

Hellman, R. P. (1981). Stability of individual loudness functions obtained by magnitude estimation and production. Perception \& Psychophysics, 29, 63-70.

JoHnson, D. H. (1980). The relationship between spike rate and synchrony in responses of auditory-nerve fibers to single tones. Journal of the Acoustical Society of America, 68, 1115-1122.

JoRIS, P. X., \& YIN, T. C. T. (1992). Responses to amplitude-modulated tones in the auditory nerve of the cat. Journal of the Acoustical Society of America, 91, 215-232.

Logue, A. W. (1976). Individual differences in magnitude estimation of loudness. Perception \& Psychophysics, 19, 279-280.

Marks, L. E., \& Stevens, J. C. (1968). The form of the psychophysical function near threshold. Perception \& Psychophysics, 4, 315-318.

Palmer, A. R, \& Russell, I. J. (1986). Phase-locking in the cochlear nerve of the guinea-pig and its relation to the receptor potential of inner hair cells. Hearing Research, 24, 1-15.

Patterson, R. D. (1987). A pulse-ribbon model of monaural phase perception. Journal of the Acoustical Society of America, 82, 15601586.

Patterson, R. D., Allerhand, M. H., \& Giguère, C. (1995). Timedomain modeling of peripheral auditory processing: A modular architecture and a software platform. Journal of the Acoustical Society of America, 98, 1890-1894.

Patterson, R. D., Handel, S., Yost, W. A., \& Datta, A. J. (1996). The relative strength of the tone and noise components in iterated rippled noise. Journal of the Acoustical Society of America, 100, 32863294.

Shofner, W. P., \& Yost, W. A. (1995). Discrimination of rippledspectrum noise from flat-spectrum wideband noise by chinchillas. Auditory Neuroscience, 1, 127-138.

SinGH, P. G., \& HirSh, I. R. (1992). Influence of spectral locus and F0 changes on the pitch and timbre of complex sounds. Journal of the Acoustical Society of America, 92, 2650-2661.

Stevens, J. C., \& Guirao, M. (1964). Individual loudness functions. Journal of the Acoustical Society of America, 36, 2210-2213.

Stevens, S. S. (1955). The measurement of loudness. Journal of the Acoustical Society of America, 27, 815-829.

Stevens, S. S. (1975). Psychophysics: Introduction to its perceptual, neural and social prospects. New York: Wiley.

Yost, W. A. (1996a). Pitch of iterated rippled noise. Journal of the Acoustical Society of America, 100, 511-518.

Yost, W. A. (1996b). Pitch strength of iterated rippled noise. Journal of the Acoustical Society of America, 100, 3329-3335.

Yost, W. A., Patterson, R. \& Sheft, S. (1996). A time domain description for the pitch strength of iterated rippled noise. Journal of the Acoustical Society of America, 99, 1066-1078.

Yost, W. A., Patterson, R. \& Sheft, S. (1998). The role of the envelope in processing iterated rippled noise. Journal of the Acoustical Society of America, 104, 2349-2361.

\section{NOTES}

1. No specific hearing test was given to the listeners prior to testing in this experiment. The listeners reported no known hearing problems and were participating in a variety of other psychophysical experiments at the Parmly Hearing Institute. The values of the best-fitting exponent $(\beta)$ obtained for the modification of Stevens's power law for individual listeners described in Table 2 were correlated with listener age. The correlation coefficients for the 2-, 4-, 8-, and 16-msec conditions were -.479, $-.608,-.085$, and -.314 , respectively. For $2-, 8-$, and $16-\mathrm{msec}$, the correlation coefficients were not significantly different from 0 ; only the correlation coefficient for $4 \mathrm{msec}$ was significantly different from 0 at the .05 level $\left(t=-2.76 ; t_{0.05(2), 13}=-2.16\right)$. We therefore conclude that age was not an important factor in the variability observed among listeners.

2. The cosine-phase and random-phase harmonic tone complexes are the only stimuli in the present study that have periodic waveforms. Although iterated rippled noises do generate the perception of pitch, they do not have periodic waveforms. Rather, iterated rippled noises have regularities in their waveforms, but these regularities are not repeated in a periodic manner. These regularities give iterated rippled noise a "pseudoperiodic" waveform.

3. This is not meant to imply that timbre differences do not exist among our test stimuli. Any periodic, complex sound possesses the perceptual attributes of loudness, pitch, and timbre, and interactions among these perceptual attributes do exist. Patterson (1987) has referred to the perceived difference between a cosine-phase harmonic tone complex and a randomphase harmonic tone complex as a difference in timbre. However, Patterson measured discrimination performance in listeners between cosinephase and random-phase complex tones and did not specifically measure timbre per se. Since there is no difference in the frequency components of the cosine-phase and random-phase harmonic tone complexes in the present study (see Singh \& Hirsh, 1992), we refer to this perceptual attribute as a pitch strength difference and not as a timbre difference. Timbre differences do exist among our stimuli. Specifically, IIRN stimuli contain both a tonal component and a noise component, whereas harmonic complex tones have a strong tonal component (see Patterson et al., 1996). However, the listeners were specifically instructed to assign a number to the test stimulus that was scaled relative to their perceptions of the pitch strengths of the first and third stimuli presented (see the Method section). Therefore, we argue that the systematic variations in the listeners' judgments primarily reflect variations in pitch strength across stimuli.

4. In order to evaluate the representation of our stimuli with the auditory image model (AIM), we used 64 auditory channels between 100 $10000 \mathrm{~Hz}$, which encompasses the frequency range of our stimuli. Setting the parameters of AIM to yield 64 frequency channels resulted in 25 channels between $100-1000 \mathrm{~Hz}$ and 22 channels between 3000 $10000 \mathrm{~Hz}$. The remaining channels occurred between $1000-3000 \mathrm{~Hz}$. The frequency channels between $1000-3000 \mathrm{~Hz}$ represent a region of transition in such a way that the temporal information available about the fine structure is decreasing, whereas the temporal information about the envelope is increasing. Since the channels between $1000-3000 \mathrm{~Hz}$ contain temporal information about both the fine structure and the envelope, we have eliminated this region from the AIM analysis for purposes of clarity.

(Manuscript received October 25, 2000; revision accepted for publication May 5, 2001.) 